MATEC Web of Conferences 33, 05008 (2015)

DOI: $10.1051 /$ matec conf/ 20153305008

(c) Owned by the authors, published by EDP Sciences, 2015

\title{
Magnetocaloric effect in Ni-Fe-Ga Heusler alloys with Co and Al substitutions
}

\author{
F. Tolea ${ }^{a}$, M. Sofronie, A. D. Crisan, M. Tolea, M. Valeanu \\ National Institute of Materials Physics, POB MG-7, 77125 Bucharest-Magurele, Romania
}

\begin{abstract}
The functionality of the ferromagnetic shape memory alloys is related to the martensitic and magnetic order-disorder transformations, both of which may be tailored by doping with other elements or by suitable thermal treatments, so that alloys with concomitant (or sequential but close) structural and magnetic phase transitions may be obtained. Concerning the magnetocaloric applications, it is assumed that the thin melt-spun ribbons assure a more efficient heat transfer. In the present work we investigate the influence of Co and Al substitutions on magnetocaloric effect characteristics of NiFeGa in bulk and also in ribbons prepared by melt spinning method and subjected to different thermal treatments. X-ray diffraction, differential scanning calorimetry, magnetocaloric and magnetoresistive characterizations have been performed. The results highlight the differences between the bulk and the ribbons (both as prepared and annealed) and the role of substitutions.
\end{abstract}

\section{Introduction}

Ferromagnetic shape memory alloys (FSMAs) are a new class of smart materials which exhibit the well known martensitic transformation (MT) bellow Curie temperature and their functionality is related, besides the magnetic order-disorder transition, also to a first order phase transformation [1]. The huge magnetic field induced strain (MFIS) exhibited by the Ni2MnGa alloy [2] justified the increased interest in FSMAs with Heusler disordered B2 austenite structure. Further developments indicated the off-stoichiometric Ni-Fe-Ga alloys as good candidates to replace the brittle Ni-Mn-Ga $[3,4,5]$ due to improved mechanical durability when experiencing a reversible austenite-martensite transformation.

The martensitic phase in these alloys features structural twinning, responsible for their good magnetoelastic properties. The precipitation of the secondary FCC $(\gamma)$ phase is inherently found for Ni-Fe$\mathrm{Ga}$ alloys with $\mathrm{Ga} \leq 27 \%$ at, this composition being located in the $\beta+\gamma$ two phase zone; in addition, this phase, located mainly at the grain boundaries, is responsible for the improved ductility of Ni-Fe-Ga based alloys [6]. New families of Heusler type alloys such as Ni-Mn- $(\mathrm{Ga}, \mathrm{Al}$, In, Sn) [7,8,9], Co-Ni-(Ga, Al) [10, 11], Ni-Fe-Al [12], that undergo a MT, were intensively studied and besides the magnetic shape memory, magneto-caloric effect (MCE), magneto-resistance effect (MRE), magneticfield-induced strain (MFIS) have been investigated. All these phenomena appeared in systems undergoing magnetic and structural phase transitions, simultaneously or delayed, but not very likely in the same alloy.
The MCE or adiabatic temperature change represents the effect of heating or cooling of a magnetic material by the application of a magnetic field. This intrinsic magneto-thermodynamic phenomenon is related to the magnetic entropy associated with the spin structure of an alloy and its change under application of an external magnetic field [13]. Several rare earth based systems $\left(\mathrm{Gd}_{5}(\mathrm{SiGe})_{4}, \mathrm{DyMn}{ }_{2} \mathrm{Ge}, \mathrm{Gd} \mathrm{RuGe}_{2},\right)$ exhibiting magnetic and structural phase transitions occurring in the same temperature range, have been reported to show very large MCE [14].

FSMA of the Heusler type materials exhibit simultaneously ferromagnetism and thermoelastic structural MT. As effect of coupling between the structural and magnetic transitions (magnetostructural coupling) a giant MCE is expected, promoting these alloys as potential performing magnetic refrigerant materials. Also, transport properties depending on crystal structure and phase transformations may offer another possibility for practical use of FSMAs: switching devices or actuators, displacement/force sensors, and motion dampers. Because ductility is an important issue in the application of FSMA as magnetocaloric materials, MCE in Ni-Fe-Ga alloys was investigated $[13,15,16]$. Numerous substitutions have been tested on $\mathrm{Ni}-\mathrm{Fe}-\mathrm{Ga}$ alloys in order to increase the Curie temperature, magneto-crystalline anisotropy or the mechanical properties $[6,17,18,19]$. Morito et al. [20] found that the addition of $\mathrm{Co}$ in $\mathrm{Ni}-\mathrm{Fe}-\mathrm{Ga}$ increases the magnetocrystalline anisotropy energy and the Curie temperature, while the MT is around room temperature. These findings motivated our study of different

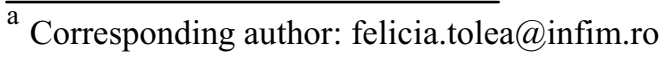

This is an Open Access article distributed under the terms of the Creative Commons Attribution License 4.0, which permits unrestricted use distribution, and reproduction in any medium, provided the original work is properly cited. Article available at nttp://www.matec-conterences.org or nttp://dx.dol.org/10.1051/mateccont/20153305008 
substitutions possibly of fundamental but also applicative interest.

In the present work, we have investigated the influence of $\mathrm{Co}$ and $\mathrm{Al}$ addition on the magnetic properties and MT characteristics of NiFeGa as bulk and also in ribbons prepared by melt spinning method and subjected to different thermal treatments. We chose $\mathrm{Ni}_{52} \mathrm{Co}_{2} \mathrm{Fe}_{20} \mathbf{G a}_{26}$ and $\mathrm{Ni}_{52} \mathrm{Fe}_{20} \mathrm{Co}_{2} \mathbf{G a}_{23} \mathrm{Al}_{3}$ alloys, prepared as bulk or ribbons, with the same valence electrons concentration $(\mathrm{e} / \mathrm{a}=7.76)$. It is known that the valence electrons concentration has a direct effect on the MT temperature [21], making it insightful to compare alloys in which the same e/a value is obtained by different substitutions, in particular in regarding the MCE and MR effects.

Reversible thermo-elastic transformation was highlighted in all samples. The Curie temperatures slightly decrease with Al addition. All investigated alloys have MT temperatures below $\mathrm{T}_{\mathrm{C}}$, so qualify as FSMAs.

From the studied samples, the most appropriate material for magnetic refrigeration turned out to be the $\mathrm{Ni}_{52} \mathrm{Co}_{2} \mathrm{Fe}_{20} \mathrm{Ga}_{26}$ alloy, prepared as ribbons and annealed at $400^{\circ} \mathrm{C}$, which shows relatively close magnetic and structural transitions near room temperature.

\section{Experimental}

Ingots of nominal composition of $\mathbf{N i}_{52} \mathbf{C o}_{2} \mathbf{F e}_{20} \mathbf{G a}_{26}$ and $\mathrm{Ni}_{52} \mathrm{Fe}_{20} \mathrm{Co}_{2} \mathrm{Ga}_{23} \mathrm{Al}_{3}$, denoted $\mathrm{Co2}$ and respectively Co2Al3, were prepared from high purity elements, by arc melting under argon protective atmosphere. Small pieces of each ingot were preserved as bulk reference and subjected to a thermal treatment in high vacuum for $25 \mathrm{~h}$ at $1223 \mathrm{~K}$, followed by a quenching in iced water. The remaining ingots were inductively melted in quarts tubes under argon atmosphere and rapidly quenched by melt spinning technique (Buhler Melt Spinning Device). The melt spun ribbons were subjected to thermal annealing: 10 minutes at $400 \mathrm{C}$ (TT1) and 2 minutes at $900 \mathrm{C}$ (TT2) followed by rapid cooling. Each samples have been tested for the MT by thermal analyses using the differential scanning calorimeter ( Netzsch DSC $204 F 1$ with the Proteus Software) in the temperatures range $80-400 \mathrm{~K}$ with different scanning rate under $\mathrm{He}$ protective atmosphere and the MT enthalpy and characteristic temperatures were evaluated. The identification of the phases and the structural study was performed by X-ray diffraction using a Bruker D8 Advantage diffractometer in the Bragg-Brentano geometry, with $\mathrm{Cu} \mathrm{K} \alpha$ radiation, at room temperature (RT).

The MT temperatures and enthalpies have been correlated with crystalline structure and microstructure on "as quenched" and after different thermal treatments to outline the special features of the MT in textured polycrystalline ribbons obtained by melt spinning. Magnetic characterization $[\mathrm{M}(\mathrm{T})$ in low and high fields; $\mathrm{M}(\mathrm{H})$ ] was done with the SQUID magnetometer in RSO mode (for $\mathrm{T}<350 \mathrm{~K}$ ); the resistivity and magnetoresistance of the samples were measured by standard four-probe method using QD-PPMS-14.

\section{Results and discussions}

\subsection{Structure}

The room temperature XRD diffractogram performed on Co2_AQ ribbons is highlighting the coexistence of the martensite phase with modulated tetragonal structure with disordered B2 austenite structure. For Co2A13_AQ ribbons, the room temperature XRD pattern shows only peaks belonging to disordered austenite B2 structure proving the martensite transformation below room temperature. Heat treatment at relatively low temperatures, $400 \mathrm{C}$, has the effect of relaxation of frozen tensions during rapid cooling process in ribbons, which is manifested by the decrease in martensitic transformation temperatures. Thus, Co2_400 alloy has at room temperature the ordered $\mathrm{L} 2_{1}$ cubic structure of Heusler alloys type specified in austenite. The thermal treatment at $900 \mathrm{C}$ has a much more dramatic effect on the MT temperature and the explanation is given by XRD patterns showing the characteristic lines of the cubic structure of austenite but in addition there is a secondary phase - $\gamma$ - face-centered cubic phase.

XRD pattern for both bulk compositions evidence, apart from the main peaks corresponding to the B2 austenite structure, some fcc $\gamma$ peaks but there are also several peaks belonging to the non modulated martensite $\left(\mathrm{L} 1_{0}\right)$. This martensite peaks are probably stress induced during sample preparation by polishing. Co2_Bulk sample evidence the austenitic B2 structure and secondary $\gamma$ FCC phase at RT.
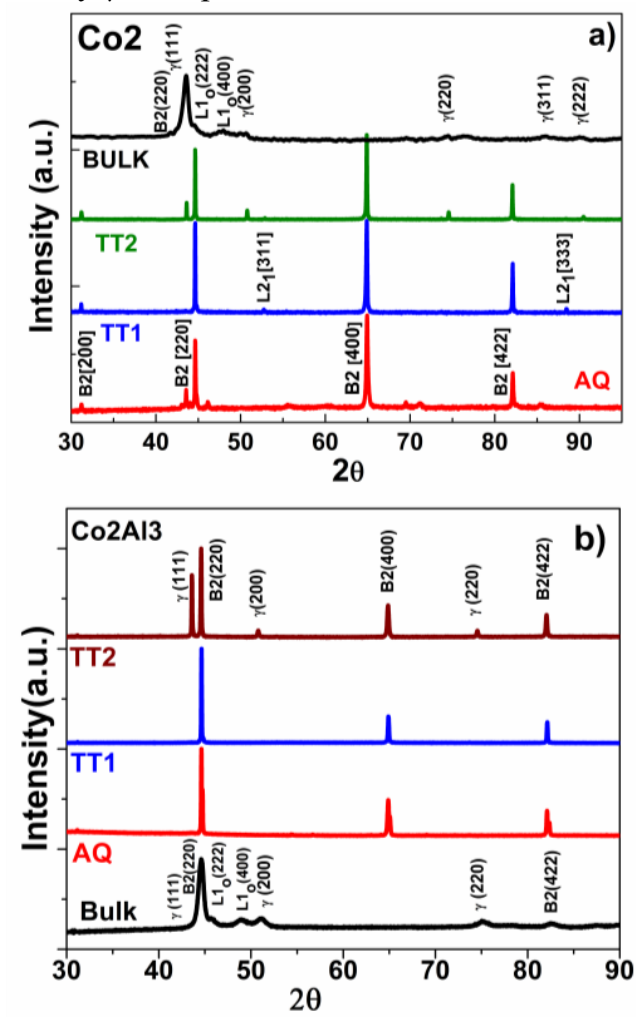
Figure 1. a) Room temperature XRD patterns of $\mathrm{Co} 2$ BULK, AQ, annealed at $400 \mathrm{C}$ and $900 \mathrm{C}$ samples ; b) the same for Co2Al3 samples.

\subsection{Calorimetry data}

DSC measurements were performed over a temperature range between $100 \mathrm{~K}$ and $400 \mathrm{~K}$. The thermoelastic transformation was evidenced on all samples. The as prepared ribbons exhibit the highest transformation enthalpy (Q). The MT characteristics, namely the forward $\left(\mathrm{T}_{\mathrm{M}}\right)$ and reverse peak $\left(\mathrm{T}_{\mathrm{A}}\right)$ temperatures and the mean value of the transformation heat (calculated as the average between the forward and reverse transformations) for the discussed alloys are given in Table 1. From the analysis of structural and DSC data results that characteristic temperatures of martensitic transformation are strongly influenced by the substitutions. Both samples shows the same 7.76 value for e/a (Fig.2a), but replacing $3 \mathrm{Ga}$ atoms with $\mathrm{Al}$, a significant decrease of the MT temperatures occurs. This is probably an atomic size effect; although the $\mathrm{Al}$ atomic radii is lower than for $\mathrm{Ga}$ the lattice constant for the $\mathrm{B} 2$ structure calculated from the XRD patterns remain unchanged for both compositions suggesting a decrease of the system energy due to a lattice relaxation and the stabilization of the austenite phase. The influence of the thermal treatments on the MT temperatures of the both compositions is illustrated in Fig. $2 \mathrm{~b}$ and Table 1.

Table 1. DSC peak transformation temperatures $\left(T_{M}\right.$ and $T_{A}$ for the direct and reverse MT respectively), the Curie temperature Tc and the average transformation heat Q (calculated as average between the direct and reverse transformation) for studied samples as bulk (Bk) or as quenched (AQ) and thermal treated ribbons (at the indicated temperature). Entropy variation $\Delta \mathrm{S}$ was evaluated only for the three samples presented in Fig. $4 \mathrm{~b}$.

\begin{tabular}{|l|l|c|c|}
\hline \multicolumn{1}{|c|}{ Sample } & $\begin{array}{l}\mathbf{T}_{\mathbf{M}} / / \mathbf{T}_{\mathbf{A}} \\
\mathbf{T c}(\mathbf{K})\end{array}$ & $\begin{array}{c}\mathbf{Q} \\
(\mathbf{J} / \mathbf{g})\end{array}$ & $\begin{array}{c}\Delta \mathbf{S} \\
(\mathbf{J} / \mathbf{K g K})\end{array}$ \\
\hline Co2_Bk & $\begin{array}{l}243 / / 255 \\
345\end{array}$ & 2.6 & \\
\hline Co2_AQ & $\begin{array}{l}294 / / 308 \\
360\end{array}$ & 3.6 & \\
\hline $\begin{array}{l}\text { Co2_2m400C } \\
\text { (TT1) }\end{array}$ & $\begin{array}{l}269 / / 284 \\
370\end{array}$ & 2.6 & -2.2 \\
\hline $\begin{array}{l}\text { Co2_2m900C } \\
\text { (TT2) }\end{array}$ & $\begin{array}{l}226 / / 250 \\
340\end{array}$ & 2.14 & \\
\hline Co2A13_Bk & $220 / / 230$ & 1.3 & \\
\hline Co2A13_AQ & $\begin{array}{l}248 / / 262 \\
350\end{array}$ & 2.5 & -1.6 \\
\hline $\begin{array}{l}\text { Co2A13_20m } \\
\text { 400 (TT1) }\end{array}$ & $\begin{array}{l}244 / / 259 \\
365\end{array}$ & 2.74 & -0.86 \\
\hline $\begin{array}{l}\text { Co2A13_20m } \\
\text { 900 (TT2) }\end{array}$ & $\begin{array}{l}181 / / 150 \\
340\end{array}$ & 0.15 & \\
\hline
\end{tabular}
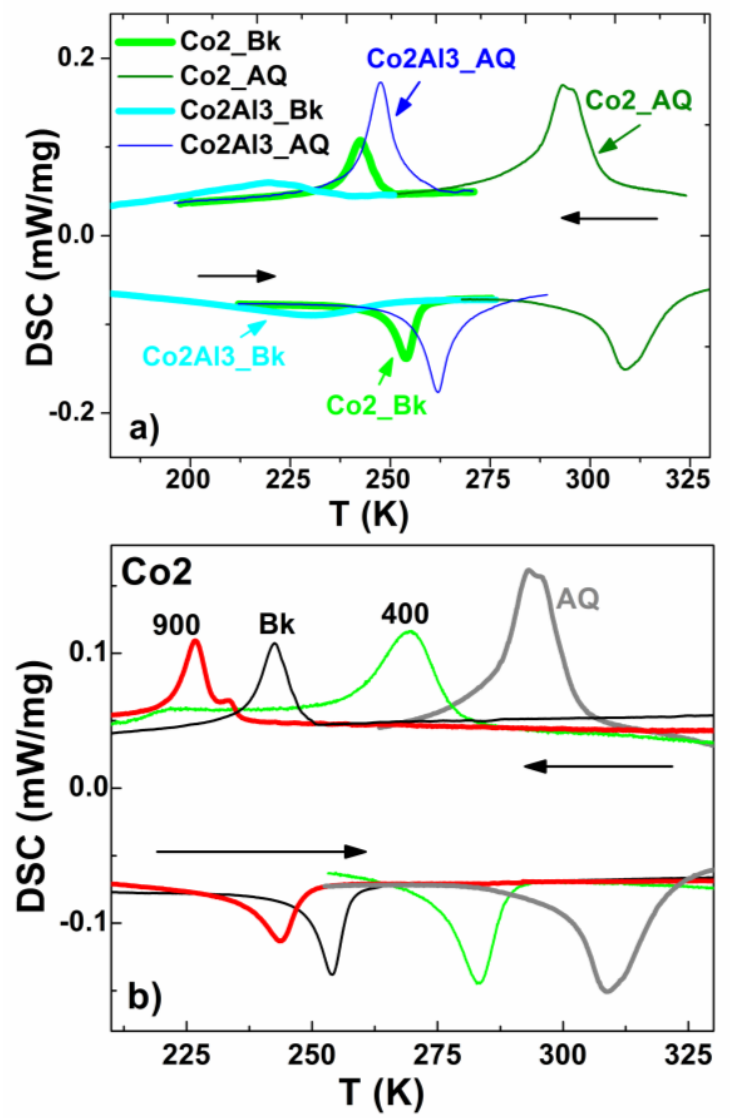

Figure 2. a) The DSC curves for Bulk and AQ ribbons for $\mathrm{Co} 2$ and $\mathrm{Co} 2 \mathrm{Al} 3$ samples. b) The martensitic transition temperatures are strongly influenced by the thermal treatments as is illustrated for $\mathrm{Co} 2$ samples.

\subsection{Thermomagnetic data}

With respect to the magnetic behavior of $\mathrm{Co} 2$ alloy, AQ and thermal treated ribbons, Fig.3a shows the temperature dependence of magnetization, measured in low magnetic field. Comparatively to the AQ alloys, the Curie temperature for annealed at $400 \mathrm{C}$ ribbons is slightly higher reflecting a higher atomic order and the relaxation of the quench-in strains induced by the processing route.

Interestingly however, for both compositions, the thermal treatment at $900 \mathrm{C}$ does not produce a further increase of the magnetic ordering temperature (Tc). The effect of further structural refinement and lattice relaxation is counteracted, as much as to a decrease, by the precipitation of the secondary $\gamma$ phase, which depletes the matrix in $3 \mathrm{~d}$ elements, inducing also a decrease of Tc [22]. 

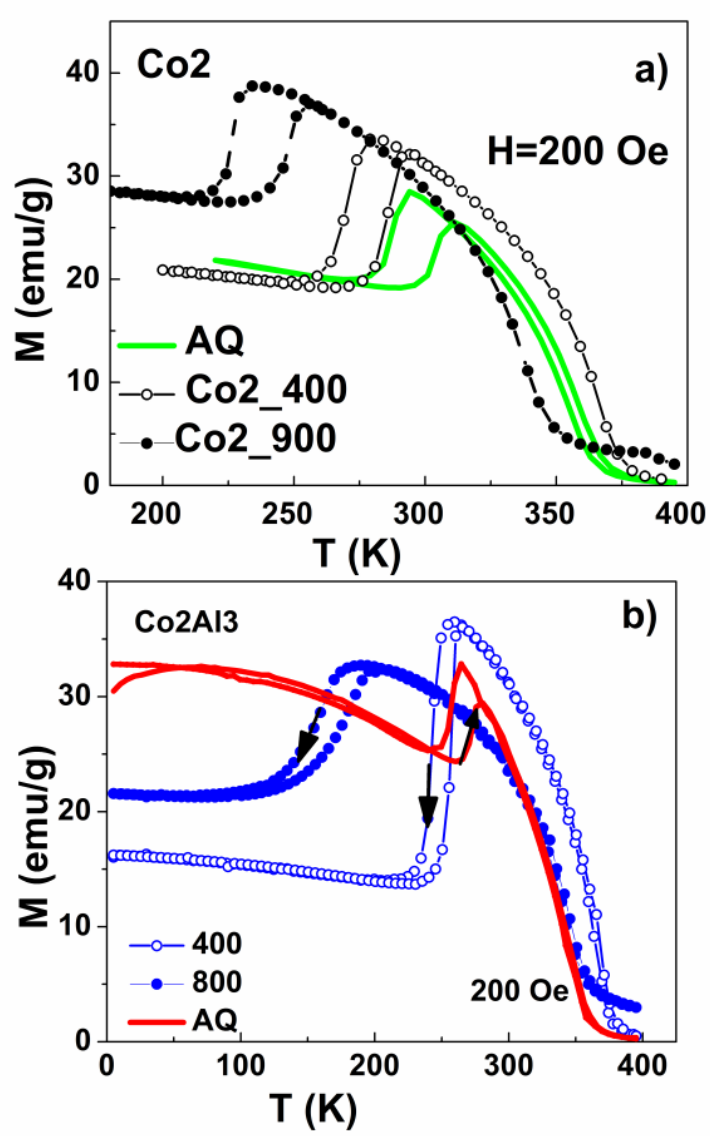

Fig.3 a) The Martensitic transformation reflected by the thermomagnetic scans at low magnetic fields on $\mathrm{Co} 2$ samples; b) the same in $\mathrm{Co} 2 \mathrm{Al} 3$

Residual magnetization is observed on Co2-900 and Co2Al3-900 even for $\mathrm{T}>\mathrm{Tc}$ (of the main phase) proving a higher Curie temperature of the FCC phase. By comparing DSC and thermo-magnetic results, the Co2A13 AQ ribbons exhibit consecutive but close magnetic and structural transitions (Fig.3b). Materials with concomitant structural and magnetic transitions are expected to have a large entropy variation and be attractive by an important magnetocaloric effect.

\subsection{Magnetocaloric effect evaluation}

To highlight the magnetocaloric effect, we choose the $\mathrm{Co} 2$ sample annealed for $10 \mathrm{~min}$ at $400^{\circ} \mathrm{C}$, with $\mathrm{MT}$ temperatures presented in Fig.3a in a range of temperature $(<380 \mathrm{~K})$ that allowed full characterization of magnetic alloy with SQUID device.

In order to quantitatively characterize the MCE, an indirect technique to evaluate the $\Delta \mathrm{Sm}$ was used. According to Maxwell relation, $\Delta \mathrm{Sm}$ can be calculated by means of magnetic measurements:

$$
\Delta \operatorname{Sm}(T, H)=\int_{0}^{H}(\partial M / \partial T)_{H} d H,
$$

whose discrete version (for isotherms measured at certain field only) reads:

$$
\Delta S m=\sum_{n} \frac{\left(M_{n+1}-M_{n}\right)}{\left(T_{n+1}-T_{n}\right)} \Delta H_{n}
$$

Magnetization isotherms in 4T maximum applied field (measured with $5 \mathrm{~K}$ temperature increment) (inset Fig.4a) and also isofield magnetization curves at different magnetic fields (Fig. 4a) were used to evaluate the magnetocaloric effect. Entropy variations versus temperature are shown in Figure $4 \mathrm{~b}$.
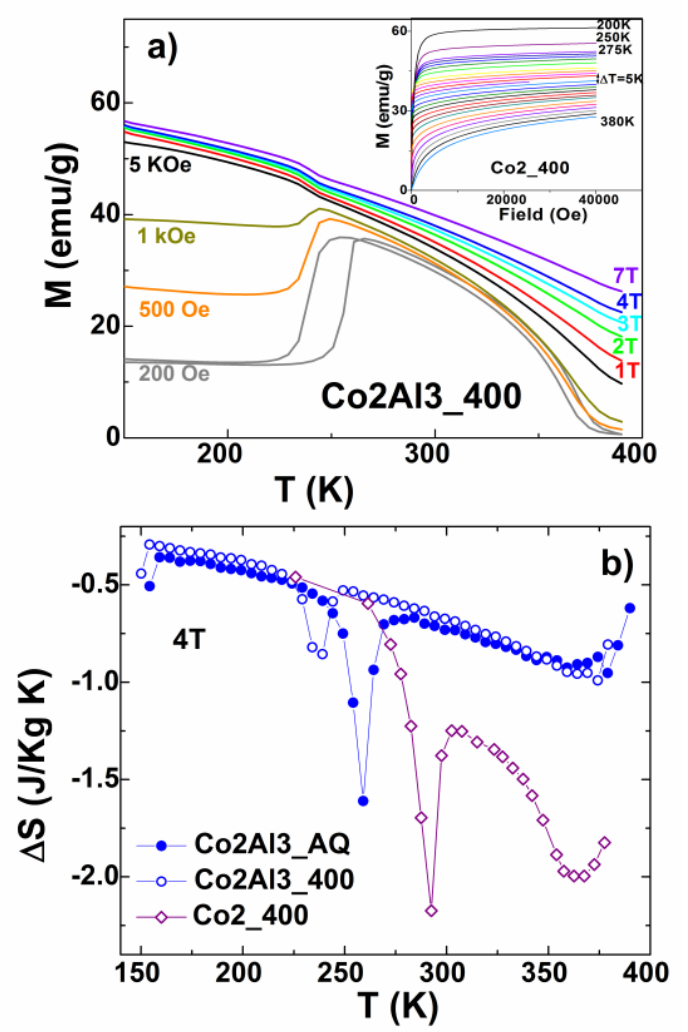

Fig.4 a) Temperature variation of the magnetization on Co2Al3 400 in different magnetic fields; Inset: magnetization isotherms in 4T for Co2_400 sample b) Entropy variation versus temperature.

For annealed (at $400 \mathrm{C}$ ) $\mathrm{Co} 2$ sample, note the occurrence of two maximum magnetic contributions to entropy, the left one in the area of martensitic transformation and the second in the order-disorder magnetic transition temperature ranges. The change in entropy is higher in the MT area whereas the refrigerant capacity (related to the peak area) is higher in Tc area. We have obtained a value of $2.2 \mathrm{~J} / \mathrm{KgK}$ for the entropy change in $\mathrm{Co} 2$ alloy, but estimate that by further tayloring the alloy compozition and using proper thermal treatments to tune the magnetic and structural transition temperatures closer (or even concomitent) this value may be improved.

\subsection{Magnetorezistive effect}

To characterize the magnetoresistance in the martensitic transformation range, we actually measured the temperature variation of the resistivity in zero and $5 \mathrm{~T}$ 
magnetic fields (inset Fig 5a) and also the continuous variation of the resistivity with magnetic field at two fixed temperatures, first in the martensite and the second in the austenitic phase for the as prepared and annealed Co2Al3 samples (Fig5b).
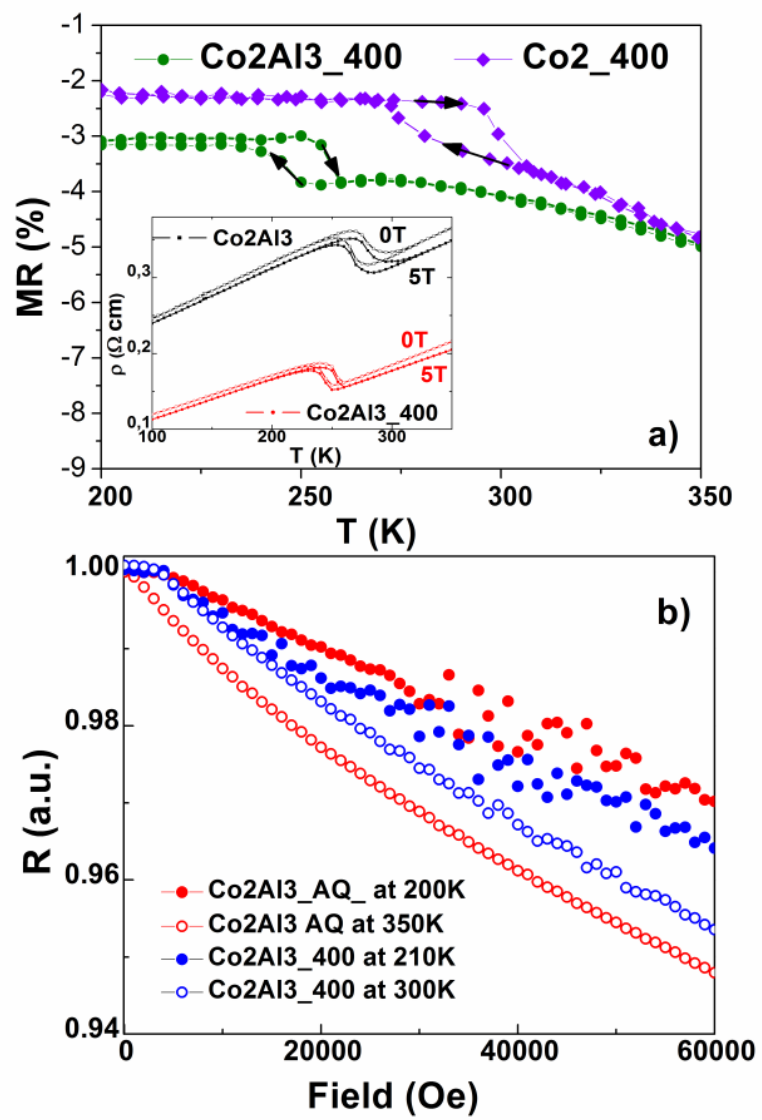

Fig.5a) The temperature variation of the magnetiresistivity for samples annealed at $400 \mathrm{C}$. Inset: resistivity variation with temperature in zero and $5 \mathrm{~T}$; b) the variation of resistance with magnetic field for Co2Al3 samples AQ and annealed at $400 \mathrm{C}$, respectively. For each sample, two temperatures are chosen, one in the martensite $(200 \mathrm{~K} / 210 \mathrm{~K})$ and the second corresponding to austenite $(350 \mathrm{~K} / 300 \mathrm{~K})$.

Thermo-resistivity measurements in different constant fields show a hysteretic character at MT which is typical for the ferromagnetic Heusler alloys. Resistivity has a metallic feature both in austenite and martensite with a jump in the passage A-M explained through increasing the scattering on boundaries variants - which do not exist in the austenite. Magnetic field leads to a slight decrease in resistivity with increasing field strength, but the effect is more pronounced in the austenitic phase probably due to the proximity of the Curie temperature of the alloy.

Magnetoresistance was calculated using the formula

$$
M R=\frac{\rho(H)-\rho(0)}{\rho(0)},
$$

The result revealed by our measurements regarding the magnetoresistive effect being greater for the austenite than in martensite is in agreement with literature data [23].
An interesting effect of non-monotonous resitance variation (with high amplitude oscillations as the magnetic field is varied) can be seen in Fig.5b for the martensite phases of $\mathrm{Co} 2 \mathrm{Al} 3$, both $\mathrm{AQ}$ and annealed at $400 \mathrm{C}$. We attribute this effect to the magnetic field effectively influencing the orientation of the martensite variants which in turn reflects in the total resistance. Changes in domains orientation (and/or domains walls) can in principle both decrease or increase the resistance leading to the observed oscillations, which are also found to be: (i) more pronounced for the AQ martensite sample (ii) starting at lower magnetic fields for the annealed sample in martensite phase and (iii) absent for the austenite samples. Results for the $\mathrm{Co} 2$ composition are similar (with less pronounced oscillations in the martensite phase, and are not given here).

\section{Conclusions}

We have studied the effect of $\mathrm{Co}$ and $\mathrm{Al}$ substitutions on the magnetocaloric and magnetorezistive properties of $\mathrm{NiFeGa}$ ferromagnetic shape memory alloys. The substitutions labeled $\mathrm{Co} 2$ and $\mathrm{Co} 2 \mathrm{Al} 3$ (see description in text) have been chosen to assure the same e/a valence electrons concentration. The alloys were prepared as bulk and also as melt-spun ribbons - an unconventional preparation route that also allows subsequent tuning of the stress release and crystallites growth by thermal treatment.

All samples presented reversible thermo-elastic transformations. As a general rule, the martensitic transformations temperatures and enthalpies decrease with increasing the temperature of the thermal treatments (on the melt-spun ribbons).

Importantly however, such tuning presents an optimum in respect to the magnetocaloric effect. The highest magnetic field induced entropy variation was found for the $\mathrm{Co} 2$ alloy annealed for 10 minutes at $400^{\circ}$ $\left(\Delta \mathrm{S}_{\mathrm{m}}=2.2 \mathrm{~J} / \mathrm{KgK}\right)$. This can be associated with the fact that the magnetic and structural transitions are in close vicinity (although we did not obtain a coincidence on it).

The magnetoresistive measurements showed a nonmonotonic variation of the martensite samples resistivity with the magnetic field, possibly due to realignment of the martensite variants. Also, the MR effect is higher for the austenite than the martensite for all samples.

\section{Acknowledgements}

This work was supported by grants of the Ministry of National Education, CNCS - UEFISCDI, project number PN-II-ID-PCE-2012-4-0516 and by the Core Program $45 \mathrm{~N}$. 


\section{References}

1. V.A. Chernenko, E. Cesari, V. V. Kokorin, I. N. Vitenko, Scr. Metall. Mater. 33, 1239 (1995)

2. K. Ullakko, J. K. Huang, C. Kantner, R. C. O'Handley, V. V. Kokorin, Appl. Phys. Lett. 69, 1966 (1996)

3. K. Oikawa, Y. Ota, T. Ohmori, Y. Tanaka, H. Morito, R. Kainuma, K. Fukamichi, K. Ishida, Appl. Phys. Lett. 81, 5201 (2002)

4. R. Santamarta, J. Font, J. Muntasell, F. Masdeu, J. Pons, E. Cesari, J. Dutkiewicz, Scr. Mater. 54, 1105 (2006)

5. F.Alvarado-Hernández, D.E. Soto-Parra, R. Ochoa-Gamboa, P.O. Castillo-Villa, H. FloresZúňiga, D. Ríos-Jara, J.All.Comp 426, 442 (2008)

6. J.Liu, N. Scheerbaum, D. Hinz, O. Gutfleisch, Acta Mat, 56, 3177 (2008)

7. A. Sozinov, A.A. Likhachev,N. Lanska, K. Ullako, Appl. Phys. Lett. 80, 1746 (2002)

8. L. Manosa, A. Planes, M. Acet, E. Duman, E.F. Wassermann, J. Appl. Phys. 93, 8498 (2003)

9. Y. Sutou, Y. Imano, N. Koeda, T. Omori, K. Ishida, K. Oikawa, Appl. Phys. Lett. 85, 4358 (2004)

10. Y.X. Li, F.B. Meng, L.Q. Yan, Appl. Phys. Lett. 84, 3594 (2002)

11. S. Chatrerjee, M. Thakur, S. Giri, S. Majumdar, A.K. Deb, S.K. De, J. Alloy Compd. 456, 96 (2008)

12. S. N. Kaul, B. Annie D'Santhoshini, A.C. Abhyankar, P. Henry, Appl. Phys. Lett. 89, 093119 (2006)

13. V. Recarte, J.I. Pérez-Landazábal, C. GómezPolo, E. Cesari, J. Dutkiewicz, Appl. Phys. Lett. 88, 132503 (2006)

14. V. K. Pecharsky and K. A. Gschneidner, Jr., Phys. Rev. Lett. 78, 4494 (1997)

15. V. Recarte, J.I. Pérez-Landazábal, C. GómezPolo, E. Cesari, J. Dutkiewicz, J.Mag.Mag.Mat. 310, e999 (2007)

16. H.J. Yu, H. Fu, Z.M. Zeng, J.X. Sun, Z.G. Wang, W.L. Zhou, X.T. Zu, J. All. Comp. 477, 732 (2009)

17. W.H. Wang, G.H. Wu, J.L. Chen, C.H. Yu, S. X. Gao, W.S. Zhan, Z. Wang, Z.Y. Gao, Y.F. Zheng and L.C. Zhao, Appl. Phys. Lett. 77, 324 (2000)

18. Z.H. Liu, H. Liu, X.X. Zhang, X.K. Zhang, John Q. Xiao, Z. Y. Zhu, X.F. Dai, G.D. Liu, J.L. Chen, and G.H. Wu, Appl. Phys. Lett. 86, 182507 (2005)

19. S. K. Sarkar, A. Biswas, P.D. Babu, S.D. Kaushik, A. Srivastava, V. Siruguri. M. Krishnan, J. All. Comp. 586, 515 (2014)

20. H. Morito, A. Fujita, K. Oikawa, K. Ishida, K. Fukamichi, R. Kainuma, Appl. Phys. Lett. 90, 062505 (2007)

21. M. Sofronie, F. Tolea, V. Kuncser, M. Valeanu, J. Appl. Phys. 107, 113905 (2010)
22. F. Tolea, ; M. Sofronie, A.D. Crisan, M. Enculescu, V. Kuncser, M. Valeanu, J. All. Comp. 650, 664-670 (2015)

23. J. M. Barandiarán, V. A. Chernenko, P. Lázpita, J. Gutiérrez, and J. Feuchtwanger, Phys. Rev. B 80, 104404 (2009) 